\title{
Grain boundary passivation with triazine-graphdiyne to improve perovskite solar cell performance
}

\author{
Siqi Chen ${ }^{1,2 \dagger}$, Qingyan $\mathrm{Pan}^{2 \dagger}$, Jiangsheng $\mathrm{Li}^{1}$, Chengjie Zhao ${ }^{1}$, Xin Guo ${ }^{3}$, Yingjie Zhao ${ }^{2^{*}}$ and \\ Tonggang Jiu ${ }^{1 *}$
}

\begin{abstract}
Detrimental defects on perovskite grain boundaries (GBs) are critical factors that lead to non-radiative recombination and hysteresis. In this work, triazine-graphdiyne (Tra-GD), a nitrogen-rich two-dimensional (2D) material, was incorporated into the active layer of perovskite to modify the GBs. Tra-GD was found to distribute evenly over the bulk of the perovskite and has a strong interaction with the $\mathrm{Pb}^{2+}$ exposed at GBs, which enables it to effectively passivate GB defects and prevent ion migration. The results of Kelvin probe force microscopy and photoluminescence studies proved that the highly conjugated Tra-GD located at GBs could promote charge extraction and transport. Benefiting from defect passivation and more efficient carrier transport, the Tra-GD based device showed less non-radiative recombination loss. Consequently, the resultant device presented negligible hysteresis and yielded a high power conversion efficiency (PCE) of $20.33 \%$ in the $\mathrm{MAPbI}_{3}$-based perovskite solar cell. This approach was extended to the $\mathrm{FAPbI}_{3}$ system with a PCE of $21.16 \%$. Our Tra-GD passivation strategy provides a useful approach to effectively improving the device performance and addressing hysteresis issues.
\end{abstract}

Keywords: derivate graphdiyne, perovskite solar cells, defects passivation, negligible hysteresis, grain boundary

\section{INTRODUCTION}

Because of the virtues of outstanding light-harvesting ability, long carrier life time, and high defect tolerance, perovskite solar cells (PSCs) have been regarded as the most promising next-generation solar cells, continuously exceeding the highest certified power conversion efficiency (PCE) by up to $25.2 \%[1,2]$. However, trap-assisted non-radiative recombination induces serious energy loss and prevents PSCs from approaching the ShockleyQueisser limit [3]. The polycrystalline nature of perovskite films generates large grain boundary (GB) areas in the active layer of a PSC [4-7]. Current research has indicated that detrimental defects that are located in the GBs not only act as recombination centers, but also provide channels for ion migration, severely affecting the performance [8-11].

To alleviate the issues associated with the GBs, numerous additives have been applied to passivate GB defects and significant achievements have been obtained. However, the natural spontaneous migration that occurs in perovskite GBs strongly affects the unsustainable effectiveness of the most commonly used additives [12,13]. A large number of additives also show poor conductivity, leading to deterioration in the photovoltaic parameters [14]. Triazine-graphdiyne (Tra-GD) is a kind of twodimensional (2D) carbonous material $[15,16]$ that is imbued with pyridine-like nitrogen on the highly conjugated framework $[17,18]$, enabling a tight affinity between the Tra-GD and the perovskite surface. The 2D structure of Tra-GD may also restrict its migration in the bulk of a PSC [19], promising a sustainable passivating effectiveness. The $\mathrm{sp}-\mathrm{N}, \mathrm{sp}-\mathrm{C}$, and $\mathrm{sp}^{2}-\mathrm{C}$ conjugated structure endows Tra-GD with excellent conductivity that is beneficial for charge transport [20-22]. Therefore, Tra-GD has the potential to effectively passivate any GB defects and reduce non-radiative recombination without sacrificing conductivity.

In this study, Tra-GD was introduced into a perovskite active layer to passivate the GB defects for the first time. Tra-GD was proved to have a strong interaction with under-coordinated $\mathrm{Pb}^{2+}$ at the $\mathrm{GB}$, which enables it to

\footnotetext{
${ }^{1}$ Qingdao Institute of Bioenergy and Bioprocess Technology, Chinese Academy of Sciences, Qingdao 266101, China

${ }^{2}$ Qingdao University of Science and Technology, Qingdao 266042, China

${ }^{3}$ Dalian Institute of Chemical Physics, Chinese Academy of Sciences, Dalian 116023, China

${ }^{\dagger}$ These authors contributed equally to this work.

* Corresponding authors (emails: jiutg@qibebt.ac.cn (Jiu T); yz@qust.edu.cn (Zhao Y))
} 
effectively passivate GB defects, suppressing the non-radiative recombination. Moreover, highly conjugated TraGD located at the GB promotes electron extraction and transport. Thus, the as-fabricated perovskite/Tra-GD solar cell provided an optimal PCE of $20.33 \%$ in the methylammonium lead iodide $\left(\mathrm{MAPbI}_{3}\right)$ system, and $21.16 \%$ in the formamidinium lead iodide $\left(\mathrm{FAPbI}_{3}\right)$ counterpart. Negligible hysteresis was also observed after modification with Tra-GD.

\section{EXPERIMENTAL SECTION}

\section{Materials}

Methylammonium iodide (MAI), methylammonium chlorine (MACl), formamidine hydroiodide (FAI), lead iodide $\left(\mathrm{PbI}_{2}\right)$, and 2,2',7,7'-tetrakis[N,N-di(4-methoxyphenyl)amino]-9,9'-spirobifluorene (Spiro-OMeTAD, 99.8\%) were purchased from Xi'an Polymer Light Technology Corp. (PLT). The stannic oxide $\left(\mathrm{SnO}_{2}\right)$ colloid precursor was obtained from Alfa Aesar (tin(IV) oxide, $15 \mathrm{wt} \%$ in $\mathrm{H}_{2} \mathrm{O}$ colloidal dispersion). Potassium salt of poly[3-(4-carboxylbutyl)thiophene (P3CT-K) was synthesized through the reaction of poly[3-(4-carboxylbutyl) thiophene (P3CT, $M_{\mathrm{w}}: 30-40 \mathrm{k}$, Rieke Metals) with sodium hydroxide $(\mathrm{KOH}$, molar ratio $1: 1)$ in $\mathrm{H}_{2} \mathrm{O}$ at $60^{\circ} \mathrm{C}$ under stirring. [6,6]-phenyl-C61-butyric acid methyl ester (PC61BM) was purchased from Clevious and American Dye Source Inc and used as received without further purification. The PC61BM solution was prepared by dissolving PC61BM into chlorobenzene (CB, Aldrich) at $20 \mathrm{mg} \mathrm{mL}^{-1}$ under stirring at $60^{\circ} \mathrm{C}$ overnight. The assynthesized Tra-GD was then sequentially washed in $1 \mathrm{~mol} \mathrm{~L}^{-1}$ hydrochloric acid $(\mathrm{HCl})$ solution, deionized water, and acetone. The Tra-GD was fully dried to obtain a brown powder product and the Tra-GD mother solution was prepared by dispersing the Tra-GD powder in $N$, $\mathrm{N}$-dimethylformamide (DMF) and sonicating for over a week. The perovskite/Tra-GD precursor solution was then prepared by mixing the $\mathrm{MAPbI}_{3}$ solution with the Tra-GD solution.

\section{Fabrication of the device}

The indium tin oxide (ITO) glass substrate was cleaned by successive sonication in detergent, deionized water, acetone, and isopropyl alcohol for approximately $15 \mathrm{~min}$, and dried in a flow of nitrogen. The ITO substrates were then treated with $\mathrm{O}_{2}$-plasma for $10 \mathrm{~min}$. For the fabrication of the $\mathrm{MAPbI}_{3}$ device, P3CT-K $\left(2 \mathrm{mg} \mathrm{mL}^{-1}\right.$ in $\mathrm{H}_{2} \mathrm{O}$ ) was spin-coated onto ITO at $3000 \mathrm{rpm}$ for $60 \mathrm{~s}$. After thermal annealing in air at $150^{\circ} \mathrm{C}$ for $15 \mathrm{~min}$, the substrates were transferred into a glove box filled with $\mathrm{N}_{2}$ gas. The $\mathrm{MAPbI}_{3}$ precursor solution was prepared by dissolving $\mathrm{CH}_{3} \mathrm{NH}_{3} \mathrm{I}$ and $\mathrm{PbI}_{2}\left(1: 1,1.4 \mathrm{~mol} \mathrm{~L}^{-1}\right)$ in $1 \mathrm{~mL}$ mixed solvent of DMF and dimethyl sulfoxide (DMSO) (volume ratio 4:1). The perovskite films were fabricated by using the anti-solvent method at $5000 \mathrm{rpm}$ for $30 \mathrm{~s}$. The anti-solvent ethyl acetate $(\mathrm{EA}, 300 \mu \mathrm{L})$ was added onto the substrate $15 \mathrm{~s}$ after the initiation of the spin coating. The substrate was then placed on the hotplate at $100{ }^{\circ} \mathrm{C}$ for $15 \mathrm{~min}$ to form the perovskite films. After the substrate cooled to room temperature, the PC61BM solution $\left(20 \mathrm{mg} \mathrm{mL}^{-1}\right.$ in $\mathrm{CB}$ ) was spin-coated onto the perovskite films at $2000 \mathrm{rpm}$ for $30 \mathrm{~s}$. The zinc oxide $(\mathrm{ZnO})$ solution $\left(12 \mathrm{mg} \mathrm{mL}^{-1}\right.$ in trifluoroethanol) was then spin-coated onto the PC61BM films at $4000 \mathrm{rpm}$ for $30 \mathrm{~s}$, after which the substrate was transferred into a vacuum chamber. Thereafter, $100 \mathrm{~nm}$ of aluminium was deposited by thermal evaporation using a metal shadow mask.

For the fabrication of the $\mathrm{FAPbI}_{3}$ device, the substrate was spin coated with a thin layer of $\mathrm{SnO}_{2}$ nanoparticle film (2.67 wt\%, diluted with water) at $4000 \mathrm{rpm}$ for $30 \mathrm{~s}$, and annealed in air at $150^{\circ} \mathrm{C}$ for $15 \mathrm{~min}$. The $\mathrm{FAPbI}_{3}$ precursor solution was prepared by dissolving $1.59 \mathrm{~mol} \mathrm{~L}^{-1} \mathrm{FAI}$ and $\mathrm{PbI}_{2}$ in $1 \mathrm{~mL}$ mixed solvent of $\mathrm{DMF}$ and DMSO (volume ratio 4:1), after which $30 \mathrm{~mol} \%$ $\mathrm{MACl}$ was added to stabilize the $\alpha$-phase of the $\mathrm{FAPbI}_{3}$. The perovskite films were fabricated by using the antisolvent method at $4000 \mathrm{rpm}$ for $30 \mathrm{~s}$. The anti-solvent diethyl ether $(800 \mu \mathrm{L})$ was added to the substrate $10 \mathrm{~s}$ after the initiation of the spin coating. The substrate was then placed on the hotplate at $150^{\circ} \mathrm{C}$ for $15 \mathrm{~min}$ to form the perovskite films. The hole transfer materials were deposited by preparing Spiro-OMeTAD (Lumtech) in CB $\left(90 \mathrm{mg} \mathrm{mL}^{-1}\right)$ and mixing with $35.5 \mu \mathrm{L}$ 4-tert-butylpyridine (TBP), $23 \mu \mathrm{L}$ Li-bis(trifluoromethanesulfonyl) imide (Li-TFSI) $\left(520 \mathrm{mg} \mathrm{mL}^{-1}\right.$ in acetonitrile), and $5 \mu \mathrm{L}$ tris(2-(1H-pyrazol-1-yl)-4-tert-butylpyridine)-cobalt(III) tris(bis(trifluoromethylsulfonyl)imide) (FK209, Lumtech) (180 $\mathrm{m} \mathrm{mL}^{-1}$ acetonitrile). Finally, $80 \mathrm{~nm}$ of silver counter electrode was deposited onto the substrate by thermal evaporation.

\section{RESULTS AND DISCUSSION}

\section{Characteristics and dispersion of Tra-GD}

Tra-GD nanosheets were prepared by liquid/liquid interfacial synthesis as depicted in Fig. 1g, in which 2,4,6triethynyl-1,3,5-triazine was used as a monomer to form uniform ultrathin films via the coupling reaction [16]. Scanning electron microscopy (SEM) and atomic force 

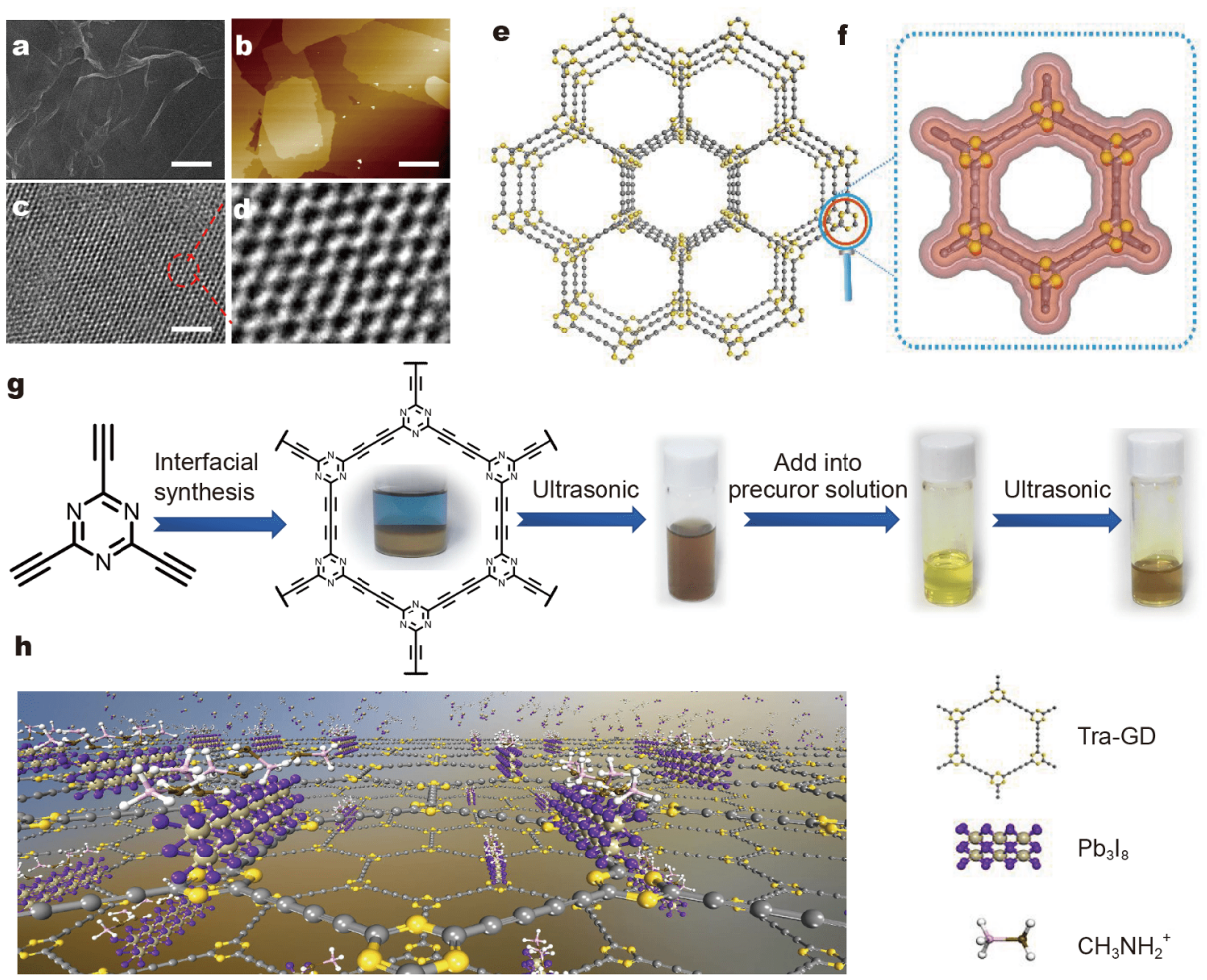

Figure 1 (a) SEM, (b) AFM, (c, d) HRTEM images of Tra-GD synthesized by the interfacial method, the scale bar is $200 \mathrm{~nm}, 4 \mu \mathrm{m}$, and $50 \mathrm{~nm}$, respectively. (e) Periodic structure of Tra-GD. (f) Structural unit of Tra-GD. (g) The preparation process of the PVSK/Tra-GD hybrid solution. (h) Schematic illustration of the PVSK/Tra-GD hybrid solution.

microscopy (AFM) were then performed to investigate the morphology of the Tra-GD. Fig. la and b show the flat plane and thin morphology of the Tra-GD films, presenting a thickness of approximately $2 \mathrm{~nm}$. The results of the high-resolution transmission electron microscopy (HRTEM) in Fig. 1c verify the orderly lattice fringe of Tra-GD. Fig. 1d is a partially enlarged view of the lattice fringe, indicating the periodic topological structure of Tra-GD. The images in Fig. 1e and $\mathrm{f}$ reveal that Tra-GD is characterized by regularly arranged $\mathrm{sp}$ and $\mathrm{sp}^{2}$ hybrid atoms that are distributed among the honeycomb-like grids. This highly ordered, conjugated structure enables efficient charge transport over the entire 2D flat plane [23].

For the preparation of the hybrid precursor solution (Fig. 1g), the as-prepared Tra-GD films were dispersed in DMF with one week of ultrasonic treatment to obtain a $10 \mathrm{mg} \mathrm{mL}^{-1}$ Tra-GD/DMF solution. The Tra-GD solution was subsequently used to prepare perovskite(PVSK)/TraGD hybrid precursor solutions at different concentrations. After sufficient heating and stirring, the precursor solutions finally turned clear without any precipitates, implying that the Tra-GD was adequately dispersed. The microscopic state in the schematic illustration (Fig. 1h) indicates that Tra-GD may have a strong affinity with plumbate colloid in the precursor solution [24].

\section{Studies of the perovskite/Tra-GD interaction}

The $\mathrm{MAPbI}_{3}$ films were prepared first (both with and without Tra-GD) and local Raman-mapping was conducted in order to examine the Tra-GD signals in the films. As the Raman signals for $\mathrm{MAPbI}_{3}$ crystal were undetectable under the excitation wavelength of $532 \mathrm{~nm}$, dim and no characteristic signals are present, as seen in the inset of Fig. 2a. However, obvious red spots are evenly distributed in the Tra-GD film (Fig. 2b) and two visible characteristic absorption peaks (G- and Y-bands) of TraGD are demonstrated in the inset, which indicates that the Tra-GD has been successfully incorporated into the $\mathrm{MAPbI}_{3}$ film with a uniform distribution. Crystalline properties were characterized via X-ray diffraction (XRD). Fig. S1 shows that the main diffraction peaks of $\mathrm{MAPbI}_{3}$ for (110), (220), and (330) remain almost unchanged and no additional diffraction peaks are seen, indicating there is no change in the lattice parameter. Thus, we conclude that the Tra-GD is probably on the 

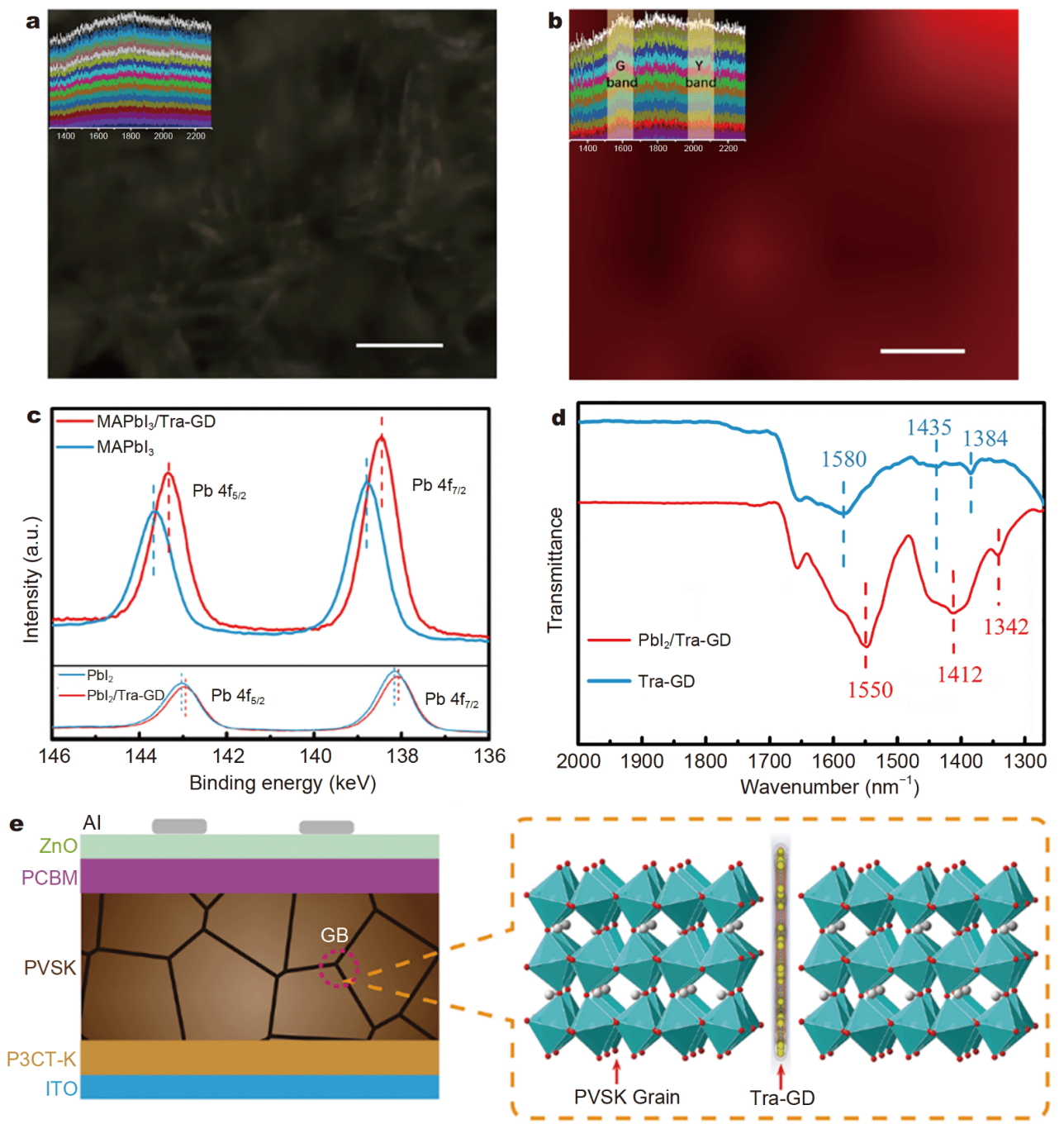

Figure 2 Raman mappings of the $\mathrm{MAPbI}_{3}$ film (a) and $\mathrm{MAPbI}_{3} / \mathrm{Tra}-\mathrm{GD}$ hybrid film (b) under $532 \mathrm{~nm}$ excitation. The scale bar is $10 \mu \mathrm{m}$. (c) XPS spectra of $\mathrm{PbI}_{2}$ and $\mathrm{MAPbI}_{3}$ with and without Tra-GD. (d) FTIR spectra of the Tra-GD and $\mathrm{PbI}_{2} /$ Tra-GD hybrid films. (e) Schematic illustration of the structure of the device (left) and Tra-GD on the GB of an active layer (right).

GBs of the perovskite rather than merged into the $\mathrm{MAPbI}_{3}$ lattice [25].

To investigate the interaction between Tra-GD and the perovskite, X-ray photoelectron spectroscopy (XPS) was performed to examine any possible links between $\mathrm{Pb}^{2+}$ and Tra-GD. As shown in Fig. $2 \mathrm{c}$, the $\mathrm{Pb} 4 \mathrm{f}$ peaks of the pure $\mathrm{MAPbI}_{3}$ are located at 138.9 and $143.4 \mathrm{eV}$. An approximately $0.4 \mathrm{eV}$ shift is observed in these signals (138.2 and $143.1 \mathrm{eV}$ ) after the incorporation of Tra-GD. Similar behavior can be observed in the $\mathrm{PbI}_{2}$ samples before and after the addition of Tra-GD. The shift of the $\mathrm{Pb} 4 \mathrm{f}$ peaks towards lower binding energies indicates an increase in the electron cloud density around $\mathrm{Pb}^{2+}$, meaning that $\mathrm{Pb}^{2+}$ may have a coordinated interaction with Tra-GD [26].

Raman spectra were then applied to analyze the interaction between the perovskite and the Tra-GD. The Dand G-bands in Fig. S2 represent the breathing vibration and stretching vibration of the triazine ring in Tra-GD, which are located at 1350 and $1570 \mathrm{~cm}^{-1}$, respectively. After the incorporation of Tra-GD, obvious variations can be observed in the D- and G-bands, indicating a strong interaction between the triazine ring in the TraGD and the perovskite [27]. Based on above analysis, Fourier transform infrared (FTIR) spectroscopy was performed to focus on the relationship between $\mathrm{Pb}^{2+}$ and the triazine ring of the Tra-GD. As shown in Fig. 2d, the characteristic peaks of Tra-GD present at 1580, 1435, and 
$1384 \mathrm{~cm}^{-1}$ can be assigned to the stretching vibrations of these triazine rings. After the introduction of $\mathrm{PbI}_{2}$, the characteristic peaks are blue-shifted to 1550,1412 , and $1342 \mathrm{~cm}^{-1}$, respectively. It is deduced that the $\mathrm{Pb}^{2+}$ draws the negative charges of the $\mathrm{N}$ atoms, resulting in a more balanced charge distribution in the $\mathrm{C}=\mathrm{N}$ bond of triazine ring. Owing to the strong interaction between $\mathrm{Pb}^{2+}$ and the triazine ring of Tra-GD, we conclude that the Pb-rich GBs in the perovskite could form a strong interaction with Tra-GD and thus ensure a tight contact between the two materials (as illustrated in Fig. 2e), which could mitigate the migration of surface ions and effectively passivate any GB defects [5].

\section{Morphology of the prepared perovskite films}

The top-view SEM and AFM images were used to analyze the dose-dependent influence of Tra-GD on crystal growth (Fig. 3a-h and Fig. S3). As the dosage of Tra-GD increases from 0 to $2 \mathrm{mg} \mathrm{mL}^{-1}$, the grain size of the perovskite is enlarged to the respective average sizes of 240, 284, and $480 \mathrm{~nm}$. In addition, the AFM images show that the perovskite film becomes smoother and the roughness is reduced from 15.3 and 15.0 , to $9.1 \mathrm{~nm}$, respectively [28-30]. Notably, when the concentration reaches $4 \mathrm{mg} \mathrm{mL}^{-1}$, a negative effect on grain growth is observed and the average size decreases to $385 \mathrm{~nm}$ and the corresponding roughness is $8.7 \mathrm{~nm}$. The correlation of grain size with Tra-GD dosage is displayed in the histograms of Fig. 3i-l, where the size distributions fit the SEM images well, indicating that a certain amount of TraGD is beneficial to the homogeneous growth of grains. We hypothesize that the interaction of pyridine- $\mathrm{N}$ with $\mathrm{Pb}^{2+}$ reduces the crystallization rate [31], leading to uniform and enlarged grains. However, an overdose of TraGD limits this process, preventing the crystals from growing larger. All these findings agree with the results of the XRD (Fig. S1) and the ultraviolet and visible spectrophotometry (UV-Vis) absorption spectra (Fig. S4), implying that the presence of Tra-GD is conducive to both grain growth and crystallinity. The enlarged grain size and better crystallinity that occur following modification with Tra-GD are beneficial for reducing the density of the defects in the active layer.

\section{Photovoltaic performance and hysteresis studies}

Devices with the structure ITO/P3CT-K/MAPbI 3 (with or without Tra-GD)/PC61BM/ZnO/Al were fabricated to examine the photovoltaic performance. According to the results of the above morphological analysis, $2 \mathrm{mg} \mathrm{mL}^{-1}$ of Tra-GD was selected as the optimal amount for addition.
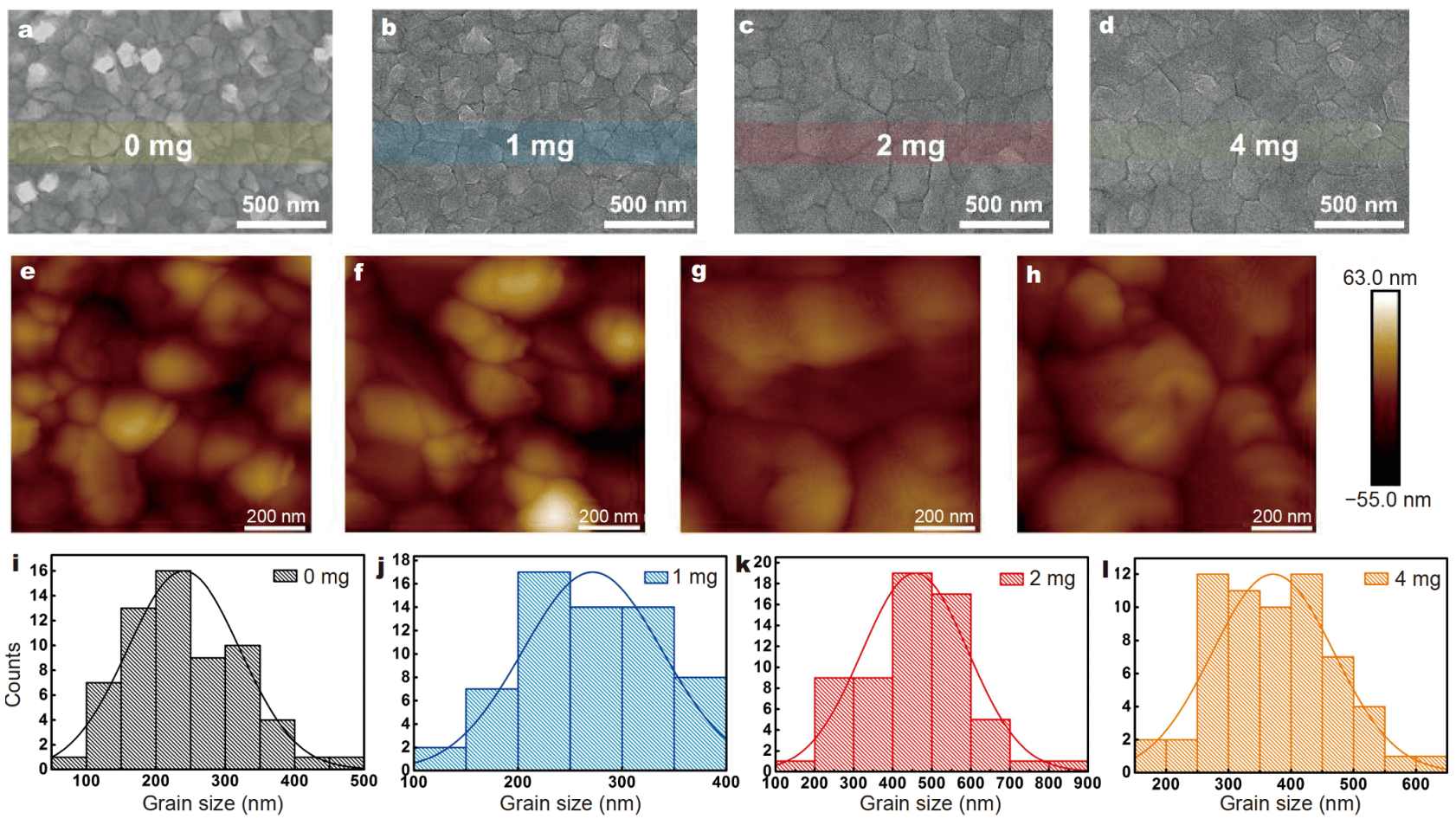

Figure 3 Top-view SEM images of the perovskite active layer with various doses of Tra-GD (a) $0 \mathrm{mg} \mathrm{mL} L^{-1}$, (b) $1 \mathrm{mg} \mathrm{mL}^{-1}$, (c) $2 \mathrm{mg} \mathrm{mL}^{-1}$, (d) $4 \mathrm{mg} \mathrm{mL}^{-1}$. (e-h) Corresponding AFM morphological images. (i-l) Corresponding grain size distributions. 
As seen in Table $\mathrm{S} 1$, the $\mathrm{MAPbI}_{3} /$ Tra-GD based device produces the best PCE at $20.33 \%$, with an open-circuit voltage $\left(V_{\mathrm{OC}}\right)$ of $1.094 \mathrm{~V}$, a short-circuit current $\left(J_{\mathrm{SC}}\right)$ of $23.3 \mathrm{~mA} \mathrm{~cm}^{-2}$, and a fill factor (FF) of $79.7 \%$, showing an impressive improvement in comparison with the control device (PCE: 17.50\%). Steady-state output PCE was performed throughout the test, the results of which indicated that the PVSK/Tra-GD based device could maintain a constant efficiency for more than $400 \mathrm{~s}$, reflecting the excellent behavior of the device under long-term testing conditions. The distributions of PCE, $V_{\mathrm{OC}}, J_{\mathrm{SC}}$ and FF are summarized as box plots in Fig. S5, which indicate that the Tra-GD based devices exhibit better performances with notable reproducibility in contrast with the control devices. As presented in Fig. 4c, the external quantum efficiency (EQE) curve of the $\mathrm{MAPbI}_{3} / \mathrm{Tra}$-GD based device is higher than its counterpart across the whole absorption spectrum, indicating a more efficient quantum conversion capability.

We also studied the stability of the device with or without Tra-GD. All the devices were stored at room temperature and the measurements were taken periodically. As shown in Fig. S6, the Tra-GD based devices retain above $90 \%$ of the original performance after more than $1100 \mathrm{~h}$ of storage, which is an obvious increase in performance compared with the control devices. The high quality of the active layer and the better crystallinity may account for this improvement in stability. Moreover, this result also confirms the sustainable effectiveness of the Tra-GD passivation.

It is notable that the introduction of Tra-GD dramatically alleviates the hysteresis of the device. Fig. 4a shows the forward-scan and reverse-scan current-voltage $(J-V)$ curves. The $\mathrm{MAPbI}_{3} / \mathrm{Tra}-\mathrm{GD}$ device shows negligible hysteresis compared with the control device. In Fig. 4b, different scan rates of 0.6, 2.4, 4.8, 7.2, 9.6 and $12.0 \mathrm{~V} \mathrm{~s}^{-1}$ were applied to detect the photocurrent-voltage relevance. The trend of the $J-V$ curves remains almost constant even when the voltage sweep rates reach $12.0 \mathrm{~V} \mathrm{~s}^{-1}$.

The alternating-current $(\mathrm{AC})$ mode $\mathrm{EQE}$ was measured under the frequencies of $30,70,110,160$, and $210 \mathrm{~Hz}$ to examine the hysteresis of the device [32]. As shown in Fig. S7, the curves of the control devices suffer from a notable deviation when the frequency is increased from 30 to $210 \mathrm{~Hz}$. Conversely, the Tra-GD based devices
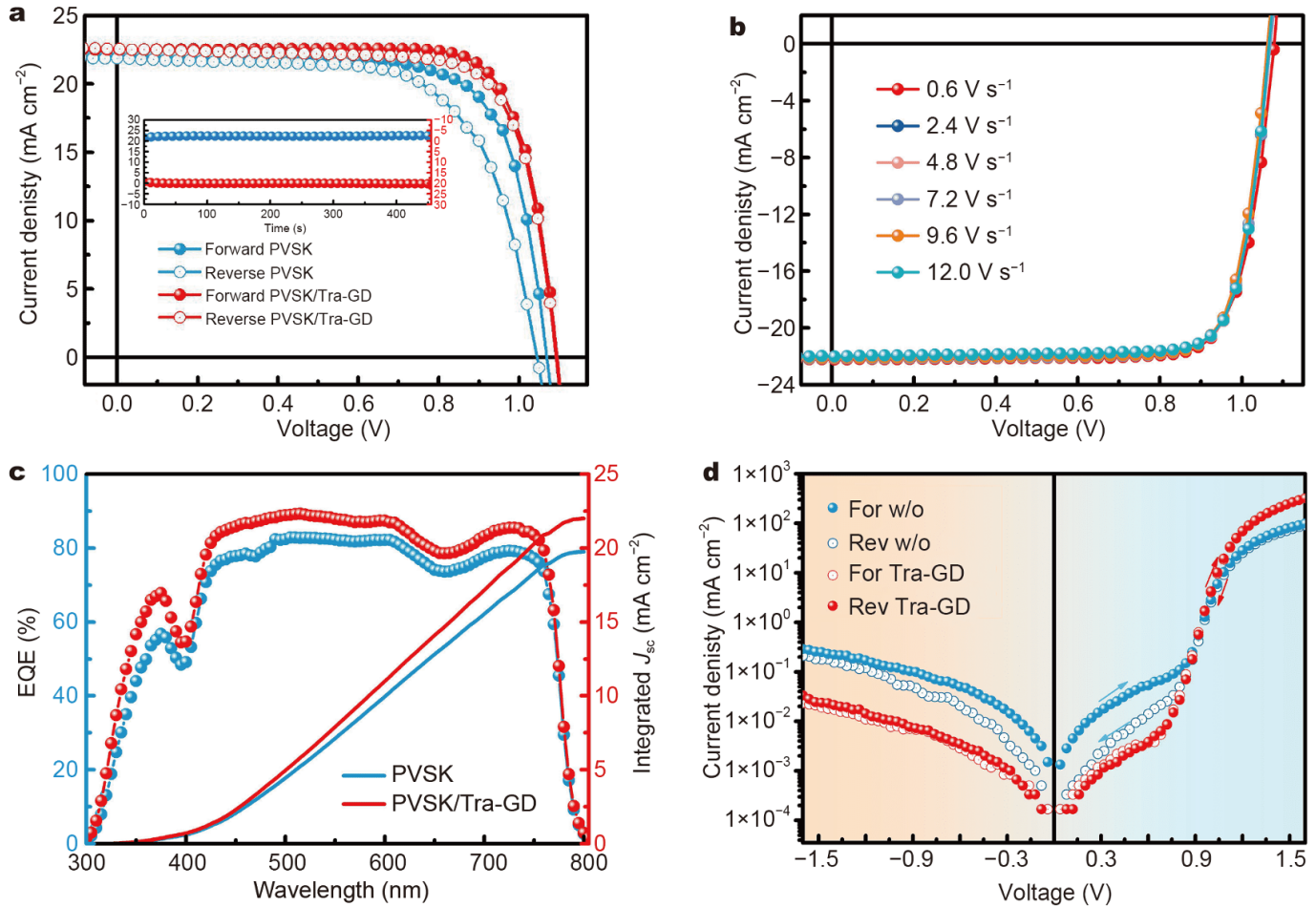

Figure 4 (a) Forward and reverse $J-V$ curves for the devices with and without Tra-GD under one-sun $\left(100 \mathrm{~mW} \mathrm{~cm}^{-2}\right)$ illumination. (b) Forward scan $J-V$ curves of the Tra-GD based device with different scanning rates of $0.6,2.4,4.8,7.2,9.6$ and $12.0 \mathrm{~V} \mathrm{~s}^{-1}$. (c) EQE spectra of the device with and without Tra-GD. (d) Forward and reverse dark $J$ - $V$ curves of the devices with and without Tra-GD. 
maintain an almost stable performance with only a small amount of hysteresis. To quantify the degree of EQE hysteresis, the EQE hysteresis index (EHI) [33] is introduced in order to calculate the discrepancy between the slowest and the fastest alternating-frequency between 30 and $210 \mathrm{~Hz}$, respectively. The value of EHI over the $300-800 \mathrm{~nm}$ range is calculated according to Equation (1),

$$
\mathrm{EHI}=\frac{\int_{300}^{800}\left(\mathrm{EQE}_{30 \mathrm{~Hz}}-\mathrm{EQE}_{210 \mathrm{~Hz}}\right) \mathrm{d} w}{\mathrm{EQE}_{210 \mathrm{~Hz}}} .
$$

The EHI value is decreased significantly from $8.66 \%$ to $0.49 \%$ after incorporating Tra-GD. The almost negligible hysteresis implies the synchronous response of the current and the alternating voltage.

The characteristics of the dark $J-V$ forward and reverse scans [34] were measured to interpret the mechanism causing hysteresis. The control device (blue curve) shows severe hysteresis compared with the Tra-GD based device (Fig. 4d). Based on previous studies [35], the ionic nature of perovskite allows the easy migration of ions through the GB, which is the root of hysteresis in perovskite solar cells. The passivation function of Tra-GD implied that the ion migration through the GBs was significantly restrained [36], meaning that the hysteresis was effectively suppressed in the Tra-GD based device.

\section{Dynamics and mechanism of charge transport}

The carrier dynamics was investigated to clarify the effect of Tra-GD on charge transport. Fig. $5 \mathrm{a}$ and $\mathrm{b}$ show the results of Kelvin probe force microscopy (KPFM) of the control and the perovskite films with Tra-GD, for which all samples were prepared on ITO/electron transport

$$
\mathbf{a}
$$
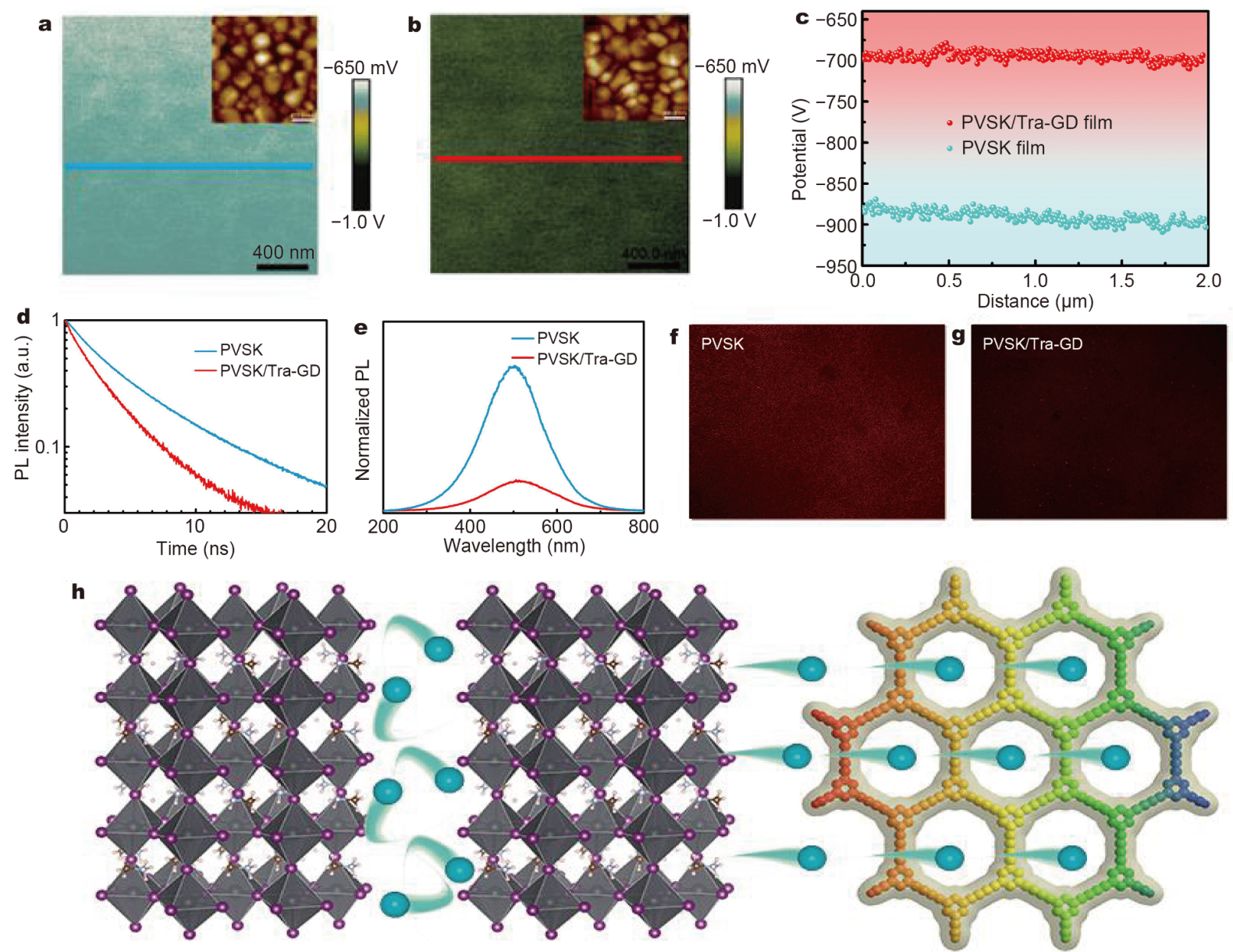

Figure 5 KPFM surface potential maps of (a) perovskite and (b) perovskite(Tra-GD) films. (c) Surface potential line profiles of the corresponding perovskite films. (d) TRPL and (e) steady-state PL spectra based on the structure of ITO/HTL/PVSK (with or without Tra-GD)/ETL. Confocal fluorescence microscopy of (f) control perovskite film and (g) PVSK/Tra-GD film. (h) Schematic illustration of Tra-GD assisting electron transport. 
layer (ETL) substrate. Images representing the corresponding surface topography are shown at the top right of the diagrams. It should be noted that all the samples were measured under illumination with light to simulate a photophysical process. After sufficient light soaking [10], the PVSK/Tra-GD film shows lower contact potential differences (CPDs) than the control film, implying the presence of a higher surface work function on the Tra-GD based film. Hence, it could be inferred that the Tra-GD facilitates carrier transport, alleviating the accumulation of electrons in trap states on the surface [37] (as depicted in Fig. 5h). Linear profiles of the CPDs are plotted in Fig. $5 \mathrm{c}$ for quantitative comparison. The CPD value of the PVSK/Tra-GD film is comparatively lower than that of the control film because of the Tra-GD assisted charge transport.

Time-resolved photoluminescence (TRPL) was also performed to explore the impact that Tra-GD has on carrier extraction. Fig. 5d presents the TRPL spectra based on the device structures ITO/hole transport layer (HTL)/PVSK (with or without Tra-GD)/ETL. The PVSK/ Tra-GD film shows a faster quenching behavior than the control. The TRPL spectra are fitted to a biexponential decay function [38], and the decay lifetimes of the PVSK/ Tra-GD and control films are determined as 3.4 and $16.7 \mathrm{~ns}$, respectively, indicating that Tra-GD is beneficial to the photogenerated carrier extraction and transport. Moreover, in Fig. 5e, the steady-state PL spectra of the PVSK/Tra-GD film shows a faster quenching profile than the control, which agrees well with the TRPL result. The visualized carrier quenching dynamics was characterized by confocal fluorescence microscopy using the samples prepared on the ITO/HTL substrates. The brightnesses of the control film in Fig. $5 \mathrm{f}$ and $\mathrm{g}$ indicate that numerous carriers undergo radiative recombination on the surface of the perovskite. Conversely, because of the more efficient carrier transport, the PVSK/Tra-GD hybrid film appears dim with few emission sites, suggesting that the carriers have been transferred instead of becoming quenched in the surface of the perovskite. Based on the above analysis, we propose there is a charge transport pathway at the PVSK/Tra-GD junction, facilitating charge extraction and transport. This conclusion could be further supported by other studies $[39,40]$ : it appears that there is a built-in electrical field at the junction that is regarded beneficial for charge separation and transport because of the coupling between the 2D material and the perovskite. Therefore, the Tra-GD not only passivates the GB defects but also plays a role in regulating the charge transport, which could account for the considerable im- provement in the efficiency of the device.

\section{Photophysical studies}

Photophysical studies were performed to reveal the intrinsic reasons for the improvement in the performance of the device. In Fig. 6a, the exciton dissociation probability $(P)$ was deduced to investigate the exciton dissociation and charge collection ability of the Tra-GD based device [41]. The $P$ value was obtained from the ratio of the saturation photocurrent $\left(J_{\text {sat }}\right)$ to the photocurrent $\left(J_{\mathrm{ph}}\right)$. The correlation between the $P$ value and the effective voltage $\left(V_{\text {eff }}\right)$ is plotted to evaluate the exciton dissociation ability. It should be noted that the PVSK/ Tra-GD exhibits a higher $P$ value than PVSK. At $0.1 \mathrm{~V}$, the $P$ values for the Tra-GD based device and the control device are 0.8 and 0.6 , respectively. A higher $P$ value is indicative of more efficient dissociation of excitons in the Tra-GD based device. The improved carrier transport dynamics was further characterized by the $J-V$ curve measurement based on the electron only structure of ITO/Al/PVSK (with or without Tra-GD)/Al. As shown in Fig. S8, the Tra-GD based device exhibits a higher current density at the same forward bias, indicating excellent electron transport capability. This could explain the improved $J_{\mathrm{SC}}$ in the Tra-GD based device. In order to further investigate the photovoltaic characteristics, a model of a single heterojunction solar cell was used to analyze the relationship between the current density and external voltage. Taking the trap-induced recombination into account, the model was built as shown in Equation (2),

$$
-\frac{\mathrm{d} V}{\mathrm{~d} J}=\frac{A K_{\mathrm{B}} T}{e}\left(J_{\mathrm{SC}}-J\right)^{-1}+R_{\mathrm{S}},
$$

where $e$ is the electronic charge, $K_{\mathrm{B}}$ is the Boltzmann constant, and $R_{\mathrm{S}}$ denotes the series resistance. In particular, the ideality factor $(A)$ delivers important information about the recombination mechanism. The linear relationship between $\mathrm{d} V / \mathrm{d} J$ and $\left(J_{\mathrm{SC}^{-}} J\right)^{-1}$ is plotted in Fig. 6b. Generally, the slope and intercept of the fitting line determine the value of $A$ and $R_{S}$. The $R_{\mathrm{S}}$ of the control device and the Tra-GD based device are 2.0 and $1.2 \Omega \mathrm{cm}^{-2}$, respectively. Accordingly, the corresponding values for $A$ are 2.4 and 1.9. As mentioned in previous studies [31], a large $A$ is often caused by serious nonradiative recombination. The Tra-GD based device displays a smaller $A$, indicating the alleviated non-radiative recombination after Tra-GD passivation. Consequently, the $V_{\mathrm{OC}}$ and $\mathrm{FF}$ are also greatly improved.

In order to gain insight into the trap density $\left(n_{\mathrm{t}}\right)$, space charge limited current (SCLC) measurements were performed, based on the device structure ITO/PC61BM/ 

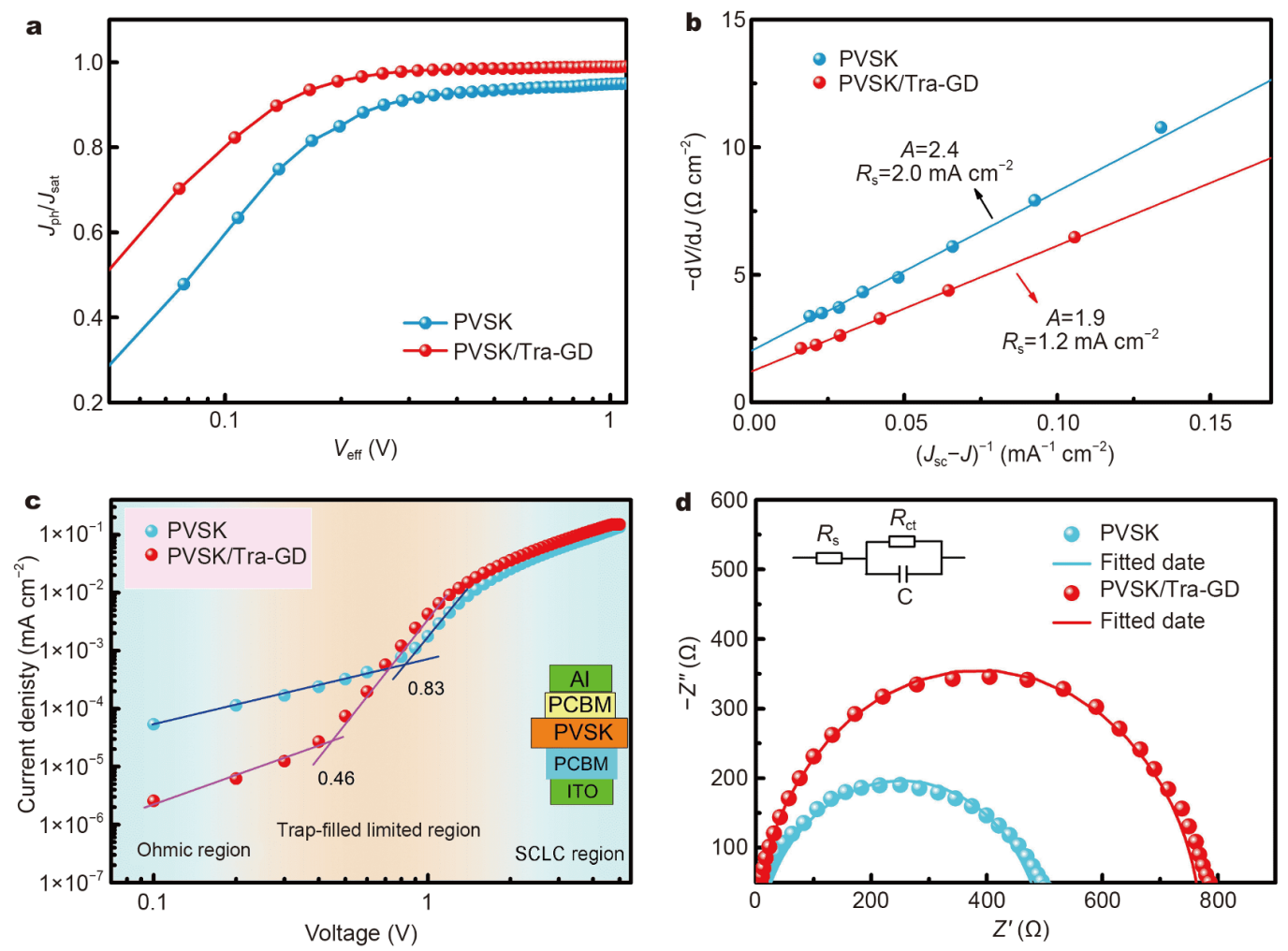

Figure 6 Based on the devices with or without Tra-GD, (a) plots of $J_{\mathrm{ph}} / J_{\text {sat }}$ with respect to $V_{\text {eff }}$ (b) plots of $\mathrm{d} V / \mathrm{d} J$ versus $\left(J_{\mathrm{sC}}-J\right)^{-1}$ and the corresponding fitting line. (c) The dark $J-V$ response of electron-only devices, with the corresponding device structure ITO/PC61BM/PVSK (with or without Tra$\mathrm{GD} / \mathrm{PC} 61 \mathrm{BM} / \mathrm{Al}$. (d) The impedance spectra (measured at $0.8 \mathrm{~V}$ bias under dark conditions).

PVSK (with or without Tra-GD)/PC61BM/Al [35]. The $n_{\mathrm{t}}$ value is calculated using the equation $n_{\mathrm{t}}=2 \varepsilon \varepsilon_{0} V_{\mathrm{TFL}} / e L^{2}$, where $\varepsilon$ and $\varepsilon_{0}$ represent the dielectric constants of perovskite and vacuum permittivity, respectively, $V_{\mathrm{TFL}}$ is the onset voltage of the trap filled limit, $L$ is the thickness of the active layer, and $e$ is the electric charge. The dark $J-V$ curves are fitted in Fig. $6 c$, producing $V_{\mathrm{TFL}}$ values of 0.83 and $0.46 \mathrm{~V}$. Based on the above equation, the $n_{\mathrm{t}}$ values of $1.835 \times 10^{16} \mathrm{~cm}^{-3}$ for PVSK and $1.017 \times 10^{16} \mathrm{~cm}^{-3}$ for PVSK/Tra-GD films are obtained. The trap density is significantly suppressed by the enlarged grain size and the passivation of the defects in the Tra-GD based device, leading to less recombination and better performance.

Electrochemical impedance spectra (EIS) were also performed to investigate the recombination of the TraGD based device. Normally, a typical PSC analogy circuit is composed of series resistance $\left(R_{\mathrm{S}}\right)$ and recombination resistance $\left(R_{\text {rec }}\right)$, which is in parallel with chemical capacitance. The value of $R_{\text {rec }}$ is associated with the diameter of the Nyquist plot semicircle, and the recombination rate could be judged by the $R_{\text {rec }}$ value. The EIS measurement was performed under dark condition and applied at a
$0.8 \mathrm{~V}$ bias voltage. The Nyquist plot (Fig. 6d) shows a bigger semicircle for the Tra-GD based device and the corresponding $R_{\mathrm{rec}}$ is $12.9 \mathrm{k} \Omega \mathrm{cm}^{2}$, which is larger than the value of control device $\left(8.3 \mathrm{k} \Omega \mathrm{cm}^{2}\right)$, indicating that non-radiative recombination is substantially restrained in the perovskite active layer as a result of the reduction in the defect density and the more efficient charge transport after Tra-GD passivation.

\section{Application in $\mathrm{FAPbI}_{3}$ based device}

The Tra-GD passivation strategy was further extended to the $\mathrm{FAPbI}_{3}$ system. The device was fabricated using the $\mathrm{ITO} / \mathrm{SnO}_{2} / \mathrm{FAPbI}_{3}$ (with or without Tra-GD)/Spiro$\mathrm{OMeTAD} / \mathrm{MoO}_{3} / \mathrm{Ag}$ structure, with the corresponding schematic illustration presented in Fig. 7a. The optimized device with Tra-GD exhibits a highest PCE of $21.16 \%$, with a $V_{\mathrm{OC}}$ of $1.132 \mathrm{~V}$, a $J_{\mathrm{SC}}$ of $24.37 \mathrm{~mA} \mathrm{~cm}^{-2}$, and an $\mathrm{FF}$ of $76.7 \%$, which is a prominent improvement compared with the control with a PCE of $18.20 \%$, a $V_{\mathrm{OC}}$ of $1.104 \mathrm{~V}$, a $J_{\mathrm{SC}}$ of $23.42 \mathrm{~mA} \mathrm{~cm}^{-2}$, and an FF of $72.9 \%$ (Fig. 7b, Table S1). Fig. $7 \mathrm{c}$ describes the negligible hysteresis in the $\mathrm{FAPbI}_{3}$-based system, in which the Tra-GD passivates the 

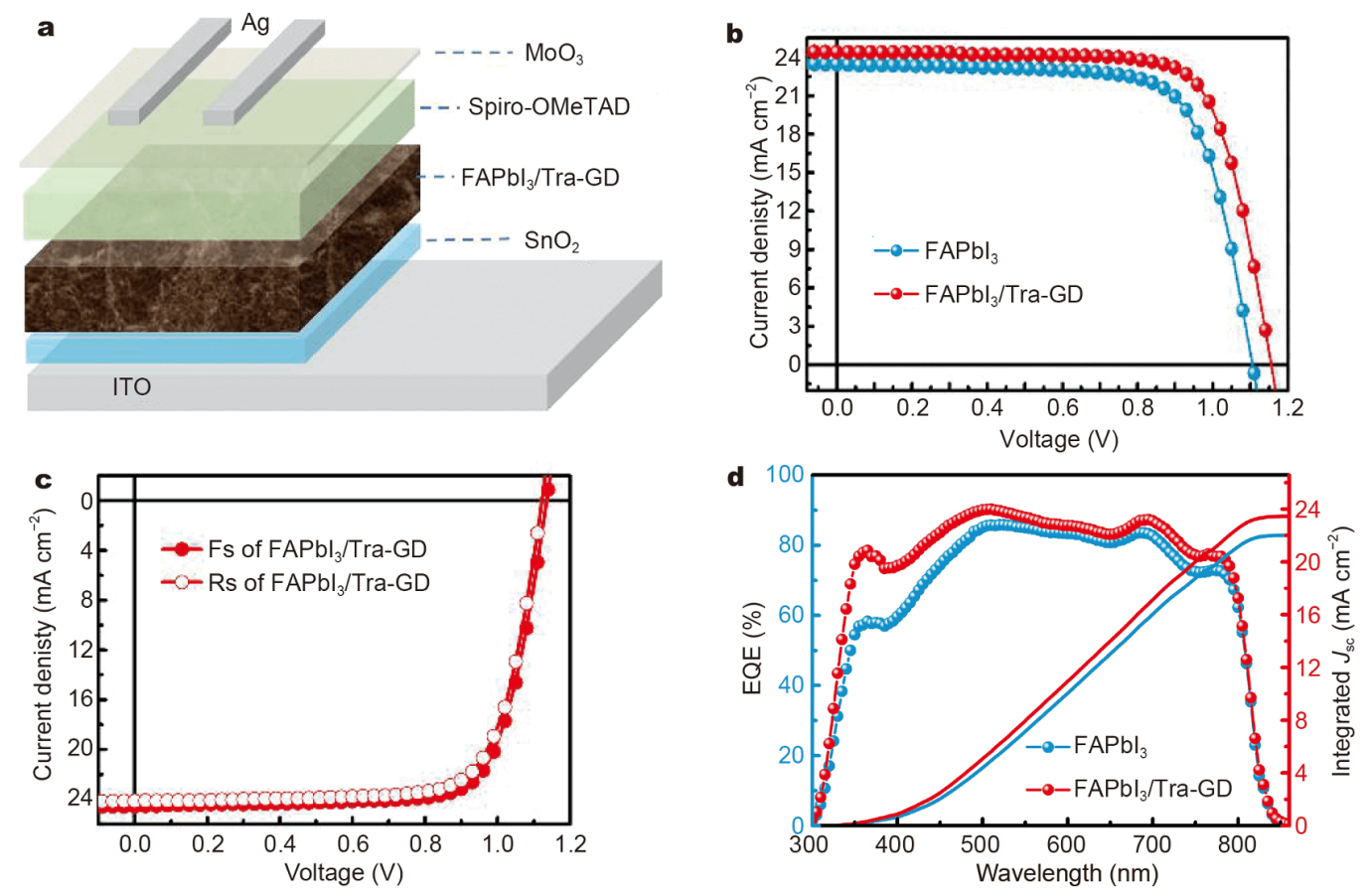

Figure 7 (a) Schematic illustration of the $\mathrm{FAPbI}_{3} / \mathrm{Tra}-\mathrm{GD}$ device structure. (b) Forward scan $J-V$ curves of the $\mathrm{FAPbI}_{3}$ based device with and without Tra-GD under one-sun $\left(100 \mathrm{~mW} \mathrm{~cm}^{-2}\right)$ illumination. (c) Forward and reverse scan $J-V$ curves of $\mathrm{FAPbI}_{3} /$ Tra-GD device. (d) EQE spectra of FAPbI based on a device with and without Tra-GD.

perovskite GBs, restricting ion mobility and reducing hysteresis. As plotted in Fig. 7d, the corresponding EQE of $\mathrm{FAPbI}_{3} /$ Tra-GD based device is effectively enhanced compared with the control, which is in agreement with the improvement in the $J_{\mathrm{SC}}$. The enhanced performance indicates that the Tra-GD passivation strategy could also be applied to the $\mathrm{FAPbI}_{3}$ system, exerting a pronounced influence.

\section{CONCLUSIONS}

In summary, we introduced the $2 \mathrm{D}$ highly conjugated carbonous material (Tra-GD) into the active layer to fabricate perovskite/Tra-GD solar cells. The strong interaction between the $\mathrm{Pb}^{2+}$ and the Tra-GD enables the Tra-GD to effectively passivate the GBs of perovskite. The presence of the Tra-GD also encourages favorable growth in the perovskite, with enlarged grain sizes and regular polycrystalline, leading to fewer GB defects. The results of the KPFM and PL studies proved that the presence of Tra-GD at GBs promotes charge extraction and transport. The photophysical studies indicated that Tra-GD effectively reduces non-radiative recombination loss in the active layer. Consequently, the as-fabricated device suffered only negligible hysteresis and yielded a peak PCE of $20.33 \%$. In addition, when Tra-GD was employed in the
$\mathrm{FAPbI}_{3}$ system, a satisfactory PCE of $21.16 \%$ was obtained. This work presents an in-depth understanding of GB passivation engineering, providing an inspiring strategy to solve the notorious problems of recombination and hysteresis in perovskite solar cells.

\section{Received 5 March 2020; accepted 1 April 2020; \\ published online 22 May 2020}

1 Best Research-Cell Efficiency from NREL. https://www.nrel.gov/ pv/assets/pdfs/best-research-cell-efficiencies. 20190923.pdf

2 Jung M, Ji SG, Kim G, et al. Perovskite precursor solution chemistry: from fundamentals to photovoltaic applications. Chem Soc Rev, 2019, 48: 2011-2038

3 Ran C, Xu J, Gao W, et al. Defects in metal triiodide perovskite materials towards high-performance solar cells: origin, impact, characterization, and engineering. Chem Soc Rev, 2018, 47: 45814610

4 Long R, Liu J, Prezhdo OV. Unravelling the effects of grain boundary and chemical doping on electron-hole recombination in $\mathrm{CH}_{3} \mathrm{NH}_{3} \mathrm{PbI}_{3}$ perovskite by time-domain atomistic simulation. J Am Chem Soc, 2016, 138: 3884-3890

5 Shao Y, Fang Y, Li T, et al. Grain boundary dominated ion migration in polycrystalline organic-inorganic halide perovskite films. Energy Environ Sci, 2016, 9: 1752-1759

6 Sherkar TS, Momblona C, Gil-Escrig L, et al. Recombination in perovskite solar cells: significance of grain boundaries, interface traps, and defect ions. ACS Energy Lett, 2017, 2: 1214-1222

7 Uratani H, Yamashita K. Charge carrier trapping at surface defects 
of perovskite solar cell absorbers: a first-principles study. J Phys Chem Lett, 2017, 8: 742-746

8 Xu J, Buin A, Ip A.H, et al. Perovskite-fullerene hybrid materials suppress hysteresis in planar diodes. Nat Commun, 2015, 6: 7081

9 Buin A, Pietsch $\mathrm{P}, \mathrm{Xu}$ J, et al. Materials processing routes to trapfree halide perovskites. Nano Lett, 2014, 14: 6281-6286

10 Shao S, Abdu-Aguye M, Sherkar TS, et al. The effect of the microstructure on trap-assisted recombination and light soaking phenomenon in hybrid perovskite solar cells. Adv Funct Mater, 2016, 26: 8094-8102

11 Leijtens T, Eperon GE, Barker AJ, et al. Carrier trapping and recombination: the role of defect physics in enhancing the open circuit voltage of metal halide perovskite solar cells. Energy Environ Sci, 2016, 9: 3472-3481

12 Zhao X, Tao L, Li H, et al. Efficient planar perovskite solar cells with improved fill factor via interface engineering with graphene. Nano Lett, 2018, 18: 2442-2449

13 De Roo J, Ibáñez M, Geiregat P, et al. Highly dynamic ligand binding and light absorption coefficient of cesium lead bromide perovskite nanocrystals. ACS Nano, 2016, 10: 2071-2081

14 Qin PL, Yang G, Ren ZW, et al. Stable and efficient organo-metal halide hybrid perovskite solar cells via $\pi$-conjugated Lewis base polymer induced trap passivation and charge extraction. Adv Mater, 2018, 30: 1706126

15 Yang Z, Liu R, Wang N, et al. Triazine-graphdiyne: A new nitrogen-carbonous material and its application as an advanced rechargeable battery anode. Carbon, 2018, 137: 442-450

16 Pan Q, Liu H, Zhao Y, et al. Preparation of N-graphdiyne nanosheets at liquid/liquid interface for photocatalytic NADH regeneration. ACS Appl Mater Interfaces, 2018, 11: 2740-2744

17 Long M, Tang L, Wang D, et al. Electronic structure and carrier mobility in graphdiyne sheet and nanoribbons: theoretical predictions. ACS Nano, 2011, 5: 2593-2600

18 Huang CS, Li YL. Structure of 2D graphdiyne and its application in energy fields. Acta Phys-Chim Sin, 2016, 32: 1314-1329

19 Chen YH, Liu HB, Li YL. Progress and prospect of two dimensional carbon graphdiyne. Chin Sci Bull, 2016, 61: 2901-2912

$20 \mathrm{Li} \mathrm{Y,} \mathrm{Xu} \mathrm{L,} \mathrm{Liu} \mathrm{H,} \mathrm{et} \mathrm{al.} \mathrm{Graphdiyne} \mathrm{and} \mathrm{graphyne:} \mathrm{from} \mathrm{theoretical}$ predictions to practical construction. Chem Soc Rev, 2014, 43: 2572-2586

21 Xue Y, Li Y, Zhang J, et al. 2D graphdiyne materials: challenges and opportunities in energy field. Sci China Chem, 2018, 61: 765-786

$22 \mathrm{Li} \mathrm{G}, \mathrm{Li} \mathrm{Y}$, Liu H, et al. Architecture of graphdiyne nanoscale films. Chem Commun, 2010, 46: 3256-3258

23 Huang C, Li Y, Wang N, et al. Progress in research into 2D graphdiyne-based materials. Chem Rev, 2018, 118: 7744-7803

24 Cao J, Jing X, Yan J, et al. Identifying the molecular structures of intermediates for optimizing the fabrication of high-quality perovskite films. J Am Chem Soc, 2016, 138: 9919-9926

25 Hong $\mathrm{H}$, Zhang J, Zhang J, et al. Ultrafast broadband charge collection from clean graphene/ $\mathrm{CH}_{3} \mathrm{NH}_{3} \mathrm{PbI}_{3}$ interface. J Am Chem Soc, 2018, 140: 14952-14957

26 Li H, Zhang R, Li Y, et al. Graphdiyne-based bulk heterojunction for efficient and moisture-stable planar perovskite solar cells. Adv Energy Mater, 2018, 8: 1802012

27 Yang N, Liu Y, Wen $\mathrm{H}$, et al. Photocatalytic properties of graphdiyne and graphene modified $\mathrm{TiO}_{2}$ : From theory to experiment. ACS Nano, 2013, 7: 1504-1512

28 Qin $\mathrm{P}, \mathrm{Wu} \mathrm{T}$, Wang Z, et al. Grain boundary and interface passivation with core-shell Au@CdS nanospheres for high-efficiency perovskite solar cells. Adv Funct Mater, 2020, 30: 1908408

29 Qin P, Zhang J, Yang G, et al. Potassium-intercalated rubrene as a dual-functional passivation agent for high efficiency perovskite solar cells. J Mater Chem A, 2019, 7: 1824-1834

30 Zhu HW, Liu YH, Felix E, et al. Tailored amphiphilic molecular mitigators for stable perovskite solar cells with $23.5 \%$ efficiency. Adv Mater, 2020, 32: 1907757

31 Li J, Jiu T, Chen S, et al. Graphdiyne as a host active material for perovskite solar cell application. Nano Lett, 2018, 18: 6941-6947

32 Habisreutinger SN, Noel NK, Snaith HJ. Hysteresis index: A figure without merit for quantifying hysteresis in perovskite solar cells. ACS Energy Lett, 2018, 3: 2472-2476

33 Lee JW, Kim SG, Bae SH, et al. The interplay between trap density and hysteresis in planar heterojunction perovskite solar cells. Nano Lett, 2017, 17: 4270-4276

34 Wetzelaer GJAH, Scheepers M, Sempere AM, et al. Trap-assisted non-radiative recombination in organic-inorganic perovskite solar cells. Adv Mater, 2015, 27: 1837-1841

35 Zhang J, Xu B, Johansson MB, et al. Strategy to boost the efficiency of mixed-ion perovskite solar cells: changing geometry of the hole transporting material. ACS Nano, 2016, 10: 6816-6825

36 Craciun NI, Zhang Y, Palmaerts A, et al. Hysteresis-free electron currents in poly( $p$-phenylene vinylene) derivatives. J Appl Phys, 2010, 107: 124504

37 Song D, Cui $\mathrm{P}$, Wang $\mathrm{T}$, et al. Managing carrier lifetime and doping property of lead halide perovskite by postannealing processes for highly efficient perovskite solar cells. J Phys Chem C, 2015, 119: 22812-22819

38 Zhang M, Dai S, Chandrabose S, et al. High-performance fused ring electron acceptor-perovskite hybrid. J Am Chem Soc, 2018, 140: $14938-14944$

39 Cao YH, Deng ZY, Wang MZ, et al. Interface engineering of graphene $/ \mathrm{CH}_{3} \mathrm{NH}_{3} \mathrm{PbI}_{3}$ heterostructure for novel $\mathrm{p}-\mathrm{i}-\mathrm{n}$ structural perovskites solar cells. J Phys Chem C, 2018, 122: 17228-17237

40 Cheng HC, Wang G, Li D, et al. van der Waals heterojunction devices based on organohalide perovskites and two-dimensional materials. Nano Lett, 2016, 16: 367-373

41 You J, Yang YM, Hong Z, et al. Moisture assisted perovskite film growth for high performance solar cells. Appl Phys Lett, 2014, 105: 183902

Acknowledgements This work was supported by the Natural Science Foundation of China (51672288 and 21975273), Taishan Scholars Program of Shandong Province, Youth Innovation Promotion Association of Chinese Academy of Sciences, Dalian National Laboratory for Clean Energy (DICP QIBEBT UN201705), and Scientific Research Cooperation Foundation of Qingdao Institute of Bioenergy and Bioprocess Technology, Chinese Academy of Sciences. We also appreciate the support of Qingdao Postdoctoral Application Research Project (2018183 and 2018186).

Author contributions Chen S conducted the experiments; Pan Q synthesized the Tra-GD; Chen S, Pan Q and Guo X performed the characterizations; Chen S, Jiu T, Zhao C wrote and revised the paper; Jiu $\mathrm{T}$, Zhao Y provided the ideas; $\mathrm{Li}$ J provided the suggestions. All authors contributed to the general discussion.

Conflict of interest The authors declare that they have no conflict of interest. 
Supplementary information Experimental details and supporting data are available in the online version of the paper.

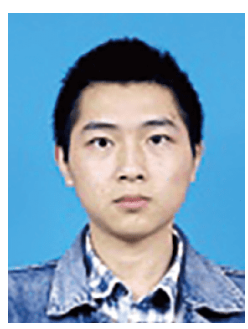

Siqi Chen received his bachelor's degree in polymer material engineering in 2017 and is currently a graduate student in Qingdao University of Science and Technology, dedicated to research of perovskite solar cell engineering.

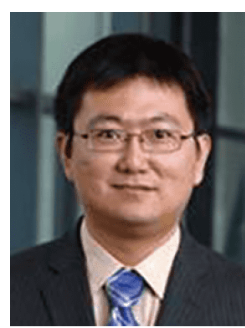

Tonggang Jiu obtained his $\mathrm{PhD}$ in chemistry from Chinese Academy of Sciences (CAS) in 2006. Currently Prof. Jiu serves as the head of carbon based energy conversion materials group at Qingdao Institute of Bioenergy and Bioprocess Technology, CAS. His research interests mainly focus on inorganic nanomaterials and solar cells based on novel 2D carbon materials of graphdiyne.
三嗪石墨炔钝化晶界缺陷以提升钙钛矿太阳能电 池的性能

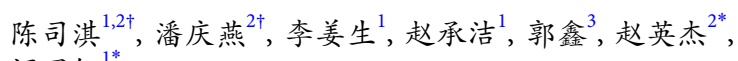
酒同钢 $^{1 *}$

摘要 钙钛矿晶界处的缺陷是造成非辐射复合和迟滞效应的主要 原因. 在本工作中, 我们将三嗪石墨炔(一种富氮的碳材料)引入到 钲钛矿中钝化晶界缺陷. 实验结果表明, 三嗪石墨炔能够均匀地分 散于钲钛矿体相中, 并且和晶界处暴露的 $\mathrm{Pb}^{2+}$ 发生强相互作用, 这 确保了三嗪石墨炔能够有效地钝化晶界, 防止晶界离子迁移. 开尔 文电镜和苂光分析证明高度共轭的三嗪石墨炔存在于晶界处, 有 利于载流子的提取和传输. 得益于晶界缺陷的针化和高效的载流 子传输, 基于三嗪石墨炔的钙钛矿太阳能电池展现了更小的非辐 射复合. 因此, 三嗪石墨炔优化后的 $\mathrm{MAPbI}_{3}$ 器件光电转换效率为 $20.33 \%$ ，迟滞效应可以忽略不计. 同样地, 将三嗪石墨炔的针化方 法拓展至FAPbI 3 器件中, 得到了 $21.16 \%$ 的最佳效率. 本文展现的三 嗪石墨炔钝化晶界缺陷的方法能够有效提升钙钛矿太阳能电池的 性能和抑制迟滞效应. 